\title{
Estudio Delphi sobre la evolución y perspectivas de la compra programática de publicidad en España
}

\section{Delphi Study on the evolution and prospects of the advertising programmatic buying in Spain}

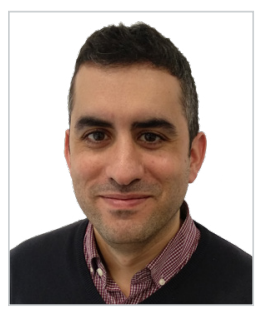

Pablo Garrido Pintado. Doctor en Ciencias de la Información por la Universidad Complutense de Madrid, con la tesis “Agencias de viajes en línea: situación y perspectivas en el comercio electrónico español”. Actualmente es profesor adjunto acreditado por la Agencia Nacional de Evaluación de la Calidad y Acreditación de la Calidad (ANECA) y responsable de innovación docente en la Facultad de Comunicación de la Universidad Francisco de Vitoria. Es autor de artículos científicos y libros sobre publicidad, marketing turístico y creación audiovisual. Es miembro activo del grupo de investigación Icono 14. Ha colaborado como profesor visitante en la Universidad de La Haya (Países Bajos) y en la Universidad San Pablo-T (Argentina).

Universidad Francisco de Vitoria, Madrid, España

p.garrido.prof@ufv.es

ORCID: 0000-0002-6111-6715

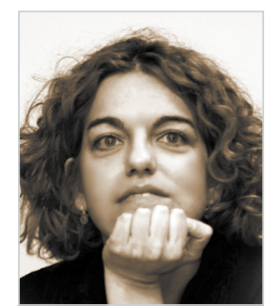

Raquel Caerols Mateo. Profesora titular, acreditada por la Agencia Nacional Española de Evaluación de la Calidad y Acreditación (ANECA), y con una reconocida experiencia en investigación de seis años. Doctora en Creatividad Aplicada por la Facultad de Bellas Artes de la Universidad Complutense de Madrid (UCM). Profesora asistente de creatividad en la Facultad de Comunicación de la Universidad Francisco de Vitoria. Ha participado como oradora invitada en conferencias internacionales como la conferencia de Arte en Computadoras, Ciudad de México o el VI Congreso de Juego y Enseñanza para una Nueva Forma de Aprender, Cartagena de Indias (Colombia). Fue co-directora del I Simposio de Cibercultura y New Media Art, financiado por el Ministerio de Cultura y Deporte de España. Universidad Francisco de Vitoria, Madrid, España r.caerols.prof@ufv.es

ORCID: 0000-0001-5167-8629

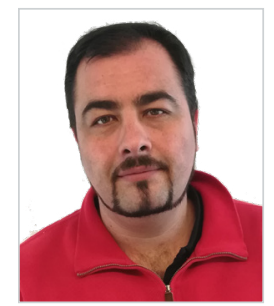

Juan Gabriel García Huertas. Profesor de la Universidad Francisco de Vitoria, donde imparte la asignatura "Gestión y desarrollo web". Doctor por la Universidad Complutense de Madrid con la tesis titulada "La Presencia del Concepto de Amistad en Facebook según el trabajo Sobre la Amistad de Pedro Laín Entralgo". Director y webmaster del departamento de fotografía de la Universidad Francisco de Vitoria. Organizador de la Semana de la Imagen de la Universidad Francisco de Vitoria y jefe de su equipo de marketing digital, y del ler Congreso Internacional SIMUFV. Ha colaborado en diversas publicaciones para congresos nacionales e internacionales. Combina su trabajo de enseñanza e investigación con el diseño de sitios web utilizando tecnología CMS.

Universidad Francisco de Vitoria, Madrid, España

jg.garcia.prof@ufv.es

ORCID: 0000-0001-5317-781X

Cómo citar este artículo:

Garrido Pintado, P.; Caerols Mateo, R. y García Huertas, J. G. (2018). Estudio Delphi sobre la evolución y perspectivas de la compra programática de publicidad en España. Doxa Comunicación, 27, pp. 253-271.

https://doi.org/10.31921/doxacom.n27a13 


\section{Resumen:}

En el presente artículo se aborda el fenómeno de la publicidad programática en España. Un sistema que automatiza el proceso de planificación de medios y mejora la eficacia y eficiencia en la compraventa de espacios publicitarios. Los objetivos principales son: establecer una definición consensuada de compra programática, conocer los nuevos roles profesionales que se demandan con el auge de esta tecnología y plantear una prospectiva sobre el futuro del sector. La técnica de investigación utilizada fue el método Delphi. Los resultados de la investigación hacen referencia a la situación, evolución y perspectivas del fenómeno, haciendo especial hincapié en falta de formación específica, falta de transparencia y desconfianza en el inventario como los principales frenos que limitan el desarrollo de esta tecnología en nuestro país.

Palabras clave:

Publicidad digital, publicidad programática, análisis de datos, perfiles profesionales, planificación de medios.

\section{Abstract:}

This article evaluates the phenomenon of programmatic advertising in Spain. Programmatic advertising automates the media planning process, and improves efficiency and effectiveness for the purchase and sale of advertising space. The main objectives of the study are: to establish consensus regarding a definition of programmatic purchase; to understand the new professional roles required as a result of this boom in technology, and to ascertain how the sector will develop in the future. The research technique used was the Delphi method. The research results relate to the current situation, evolution and perspectives of the phenomenon, with special emphasis on lack of specific training, lack of transparency and distrust in the inventory as the main obstacles that limit the development of this technology in our country.

Keywords:

Digital advertising, programmatic advertising, data analysis, professional profiles, media planning.

\section{Introducción}

La irrupción de las nuevas tecnologías ha supuesto un cambio notable en el tejido productivo afectando a la mayoría de campos profesionales y científicos.

En el ámbito de la publicidad, los primeros efectos se comenzaron a observar con el auge de la digitalización. La posibilidad de contar con software de autoedición supuso la progresiva eliminación de puestos de trabajo relacionados, en su mayoría, con la producción gráfica y audiovisual. Años más tarde, la progresiva implantación de las redes, supuso no sólo un cambio de roles dentro de la profesión, sino también, la propia redefinición del sistema publicitario. Así, para Beatriz Corredor (2011:99):

"La expansión de las redes digitales no sólo está conmocionando el panorama mediático y la búsqueda de nuevos modelos de negocio, sino que, como una correa de transmisión, está afectando a toda la cadena de valor, aspecto que implica directamente a la publicidad, tanto en su papel financiador de los viejos y nuevos medios, como a la propia esencia de la comunicación publicitaria".

Ahondando en este fenómeno, José Luis León, citado por Fajula y Roca (2000), afirma que Internet no sólo pone en crisis el concepto de publicidad, sino que plantea "una publicidad fundamentalmente híbrida, como el propio medio que la acoge".

Estamos pues en un contexto en el que la publicidad está mutando. El modelo de mensajes persuasivos dirigidos a grandes masas vira hacia el impacto de audiencias cada vez más fragmentadas. Se mezcla información, entretenimiento y publicidad, y los anunciantes, envían mensajes en un paisaje digital superpoblado de marcas, servicios y productos. Ahora la 
capacidad de atracción no se basa únicamente en el contenido, sino en la comunicación y en la relación que se crea entre usuarios y marcas. Este es el caldo de cultivo para el surgimiento de la publicidad programática, que nace como consecuencia de la automatización de compra/venta de espacios empleando modernas tecnologías.

Estas circunstancias obligan a las agencias de publicidad y medios de comunicación "a disponer de perfiles profesionales con competencias para crear, transformar y distribuir información en diversos soportes tanto tradicionales como digitales" (Álvarez-Flores, et al., 2017: 137). Favoreciendo la demanda de profesionales capaces de trabajar a distancia de forma colaborativa, no sólo gestionando diferentes plataformas sociales, sino también interpretando y gestionando grandes volúmenes de datos.

El presente trabajo parte de la siguiente hipótesis: “La compra programática supone un cambio en los roles y funciones de la agencia de medios. Su desarrollo tenderá a reconfigurar el panorama del sector, dando entrada a nuevos agentes, incentivando el surgimiento de nuevos perfiles profesionales y la desaparición de otros".

Bajo esta premisa, y con el objeto de conocer el segmento de la publicidad programática y su proyección futura, estudiaremos la evolución de la publicidad display desde sus inicios, para, finalizar, describiendo el ámbito de la compra automática de medios y audiencias. Este fenómeno cambia las reglas del juego del ecosistema publicitario modificando especialmente los roles y funciones del planificador de medios digital.

Tras el desarrollo del estado de la cuestión, aplicaremos la metodología de investigación adecuada para conseguir los siguientes objetivos:

- Proponer un consenso terminológico del concepto de publicidad programática

- Conocer los nuevos roles profesionales que surgen con el auge de la compra automatizada de espacios publicitarios.

- Estudiar la convivencia del planificador de medios digital con los nuevos perfiles que se demandan.

- Plantear una prospectiva sobre el futuro del sector

\subsection{Evolución de la publicidad display}

El 27 de octubre de 1994, la historia de la publicidad cambia para siempre. Hotwired, la versión online de Wired Magazine, publica el primer banner, un gráfico de pequeño tamaño en el que aparecía un enigmático texto: ¿Alguna vez has hecho click con tu ratón aquí? (Sánchez \& De Frutos, 2007: 160). La pieza - en la que se anunciaba la tecnológica AT\&T - era nueva e intrigante, por lo que el $44 \%$ de los que la vieron, hicieron click en ella (King, 2012: 122). Sin tener una idea aproximada del tráfico que podía atraer el portal y sin datos del perfil sociodemográfico de su audiencia, Hotwired fijó un precio de 30.000 dólares por 3 meses de publicación y, sorprendentemente, al poco tiempo, recibió solicitudes de otros anunciantes como MCI, Volvo o Sprint (Medoff \& Kaye, 2016: 72).

Craig Kanarick, uno de los consultores digitales contratados para trabajar en la campaña, recuerda que el objetivo del equipo era hacer un anuncio que no se sintiera como un anuncio (Cook, 2016) y que realmente ofreciera contenido valioso a los usuarios. "No le vendamos algo a alguien", recordó haber pensado, "vamos a recompensarlo por hacer clic en esta cosa que AT\&T le ha brindado”. 
Tal como señalan Boone, Secci y Gallant (2010), desde el diseño del primer banner hasta finales de la década de los noventa, los espacios publicitarios se vendían basándose en el coste por mil impresiones (CPM). Un método de compra poco novedoso pues, era habitual en el medio impreso (Madinaveitia, 2018). Aún así, muchos sites y portales que aglutinaban mucha audiencia, lo usaban como método de tarificación.

También a finales de los noventa surge un formato que, desde su lanzamiento, generó mucha controversia. Se trataba del pop up, definido por la Interactive Advertising Bureau como "un tipo de formato flotante que aparece en la pantalla del usuario a través de una ventana independiente o sobre los contenidos de un sitio web” (IAB, 2004:12). En un principio, se consideraron como un remedio debido al brusco descenso en los ratios de click (Chaterjee, 2008: 53; Cho, Lee y Tharp, 2001), pero pronto, comenzaron a ser una molestia (Edwards, Li \& Lee, 2001) que aceleró el desarrollo de los bloqueadores de ventanas emergentes.

A caballo entre el siglo XX y XXI se produce otro fenómeno destacable, la explosión de los motores de búsqueda. Allá por 1998, Goto.com es el primer site que lanza la subasta de publicidad por búsqueda de palabras clave. Sin embargo, aunque era un sistema novedoso en cuanto que lanza el sistema de pago por click (CPC), se percibía como poco transparente, ya que, premiaba al anunciante que invertía más por encima de la relevancia de los contenidos (Cook, 2016). Ya en el año 2000, Google lanza el programa Adwords, sistema que afina dos años después, introduciendo la asignación basada en calidad, que permite ordenar los anuncios combinando no sólo el precio de puja, sino también su popularidad (Jansen y Mullen, 2008: 119).

Sin embargo, tal y como señala la IAB (2014: 8), hasta ese momento:

"El ecosistema de compra-venta de medios era muy sencillo: los anunciantes (y algunas agencias que comienzan a posicionarse en el medio online) compraban directamente posiciones o impresiones a los soportes existentes. Pronto quedó clara una realidad evidente: la oferta superaba a la posible demanda y el inventario disponible aumentaba cada día".

Paralelamente, tras la revolución de la publicidad en buscadores, el siguiente hito viene de la mano de las redes sociales. Estas plataformas cobran fuerza durante la primera década del 2000, y rápidamente, los anunciantes se fijan en ellas pues van más allá que los medios tradicionales, ofreciendo la posibilidad no sólo de acceder a la información, sino también de compartir e interactuar de manera más efectiva. Para autores como Alhabash, Mundel y Hussain (2017:286) la publicidad en social media "es una pieza de contenido online, diseñada con una intención persuasiva y/o distribuida a través de plataformas social media que permite a los usuarios de internet acceder, participar, agregar y co-crear".

Facebook comienza a trabajar con anunciantes en 2006. Al principio, insertando enlaces (Text-links) e imágenes de pequeño tamaño. Posteriormente implementaron un sistema que permitía segmentar usuarios por características sociodemográficas e intereses.

A partir de 2010, algunas plataformas como BuzzFeed o Mashable, abren a los anunciantes nuevas opciones para conectar con la audiencia empleando contenido patrocinado y publicidad nativa (Cook, 2016). La IAB (2015:2) la define como "aquella que se integra en el contenido natural de la página (...) permitiendo a la marca estar presente en la publicación de una manera más armonizada con el resto de contenido que otros sistemas publicitarios". El formato, muy interesante 
para marcas y soportes, "complica la división entre las funciones editoriales y la publicidad, lo que genera cierta polémica" (Carlson, 2015, 158)

\subsection{La publicidad programática}

Entre 2007 y 2010 surgen plataformas de intercambio de anuncios (ad exchanges), muchas de ellas dependientes de gigantes del sector como Yahoo, Google o Microsoft, entre otros (Schmid, 2017).

Además, tal como señala la IAB (2014:9 "las agencias de medios comienzan a desarrollar capacidades y tecnologías para canalizar una buena parte de la inversión online de los anunciantes”. Precisamente, la evolución tecnológica, que amplía las posibilidades de extraer y aprovechar la información que se genera en la actividad publicitaria.

Para Gonzálvez y Mochón (2016:7), los avances tecnológicos son responsables de impulsar un nuevo paradigma donde la publicidad programática, va ganando protagonismo. Estos avances vienen representados por el aumento de la velocidad de conexión, de la capacidad computacional que permite gestionar grandes volúmenes de datos complejos, la reducción de costes, la aplicación de métodos científicos en marketing (algoritmos, modelos matemáticos, inteligencia artificial, machine learning), el uso de sistemas de reconocimiento de usuarios y el uso de procesos que permiten comprar y vender espacios publicitarios en tiempo real (Stange y Funk, 2014: 305).

Álvaro Cristóbal, Director Global de Estrategia y Operaciones de Rebold (2018) define la compra programática como

"Una disciplina del marketing digital a través de la cual se compran espacios de forma sistemática y automatizada, donde intervienen siempre dos elementos: tecnología, que nos permite automatizar el proceso de compra 24 horas al día, 7 días a la semana, y data, que permite indicar a dicha tecnología el grado de relevancia de cada impresión para tomar decisiones".

Para Nacho Carnés, profesor colaborador UFV y experto en el sector (2018), la compra programática constituye un "cambio de paradigma en la forma en la que los anunciantes compran sus medios, que conlleva la automatización de un inventario publicitario". Y añade, el programmatic ad "confiere al anunciante la capacidad de alcanzar e impactar al usuario correcto independientemente del dispositivo que utilice, en el momento adecuado, en el sitio web apropiado y utilizando un mensaje personalizado basado en el data histórico y en tiempo real". A este respecto, la data ejerce un papel absolutamente relevante, pues, tal como apuntan Malthouse, Maslowska y Franks (2018:6) "los datos están en el corazón de los enfoques computacionales. Cuantos más datos sobre individuos tengan los modelos programáticos, mejor será la orientación y personalización de los anuncios".

El ecosistema programático está formado por el lado de la demanda (compra) y la oferta (venta). Analicemos cada uno de sus componentes (IAB, 2014: 20; Carrillo-Durán y Rodríguez, Silgado, 2018:197-198): 


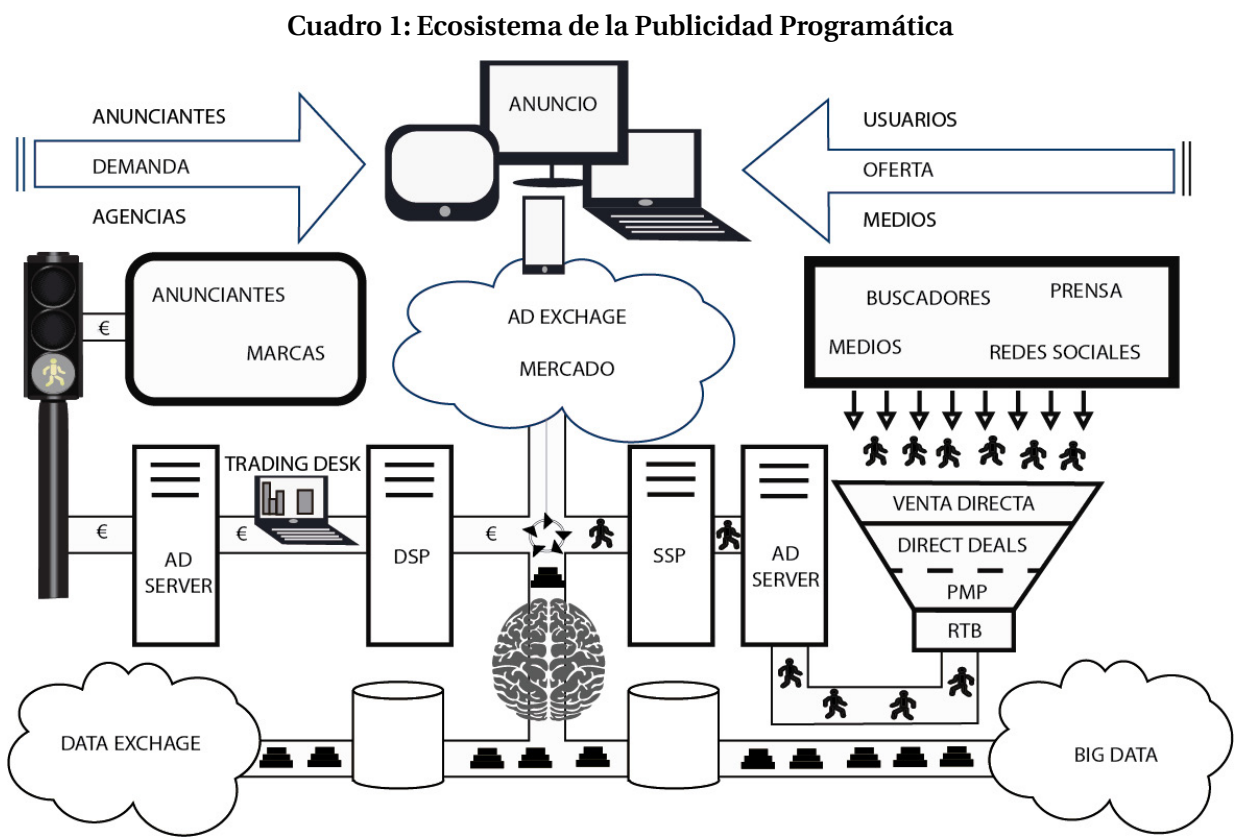

Fuente: Elaboración propia a partir de Rodríguez (2017)

- Compra (Parte de la demanda: anunciantes, agencias, traders, DSP’s, Ad exchanges)

- Anunciantes: Demandan y compran impresiones. Fijan el presupuesto que se va a emplear en la campaña, definen el público objetivo y proporcionan los materiales creativos. Suelen contratar los servicios de una agencia de medios u operar de manera directa tratando con SSPs o soportes.

- Agencias de medios: Intermediarios tradicionales en el sector. Son los responsables de la consecución de los objetivos fijados por el cliente. Gestionan el presupuesto para invertir en los DSPs, ad exchanges, redes, etc. que consideren más oportunas para el éxito de la campaña.

- Trading Desk: Conjunto de traders que preparan, lanzan y optimizan las distintas estrategias de compra de las campañas (retargeting, prospect, branding, compra específica, contextual, etc.). Pueden conectar con varios DSP’s o gestionar acuerdos cerrados con medios o redes publicitarias (Direct Programmatic). Pueden estar integrados en las agencias o trabajar de manera independiente.

- Demand Side Platform (DSP): Se trata de una tecnología de puja que permite a anunciantes y/o agencias comprar inventario en diferentes lugares de intercambio. La puja se realiza en tiempo real empleando datos de la audiencia y es conocida popularmente como Real Time Bidding (RTB). La modalidad de compra es Coste por Mil (CPM). Tal como señala Adolfo Maceiras (2018), head of traders en BMIND, siempre ha existido cierto miedo por parte de los anunciantes a comprobar dónde aparece su marca o con qué contenido va a aparecer relacionada. Por esa razón, 
la mayor parte de DSP’s están integrados con diferentes herramientas de Brand Safe. Aparte de estas herramientas, existe la posibilidad de subir al DSP una lista de sites donde queremos pujar (whitelist). Igualmente, tenemos la posibilidad de tener una blacklist donde indicamos los sites donde no queremos aparecer.

- Ad exchange: Se trata de una plataforma tecnológica que permite a un soporte definir las reglas de la puja del lado del vendedor, y al trading desk, definir las reglas del comprador así como gestionar las pujas. Pueden ser abiertos (no hay restricción de anunciantes o compradores) o privados (restringidos a unos pocos soportes y anunciantes).

- Venta (usuarios, oferta, medios...).

- Soportes: Los editores de contenidos, priorizan, a través de sus gestores de tráfico (traffickers) la venta directa. En la parte programática se trabaja a partir de acuerdos cerrados (deals), a precio fijo y con un inventario garantizado, y también, a través de un mercado privado (PMP).

- Redes Publicitarias: Funcionan de manera similar de los soportes. La principal diferencia radica en que representan a varios editores y pueden también actuar como compradores de impresiones en el ad-exchange.

- Supply Side Platforms (SSP’s): Plataformas tecnológicas ligadas a la parte de soporte (medios). Permiten acceder a fuentes y agregadores de manera automatizada. Según Paz Calvo (2017) los soportes necesitan tener esta tecnología integrada poder establecer relación con el DSP que integran agencias y anunciantes. Los SSP's necesitan, por un lado, establecer el precio mínimo de puja por el inventario (hard floor) y, por otro, fijar una serie de reglas a nivel de anunciantes: atributos técnicos, permitir o evitar ciertas categorías (ej: sexo, tabaco, etc.).

- Otros actores

- Proveedores de datos: Proveedores de datos con capacidad dirigir esos datos a las marcas y a sus acciones de medios. Las grandes plataformas de data se denominan DMP's "recolectan, integran y gestionan grandes cantidades de datos estructurados y no estructurados" (IAB, 2014:18). Los datos provienen de fuentes propias (first party data) o externas. Las fuentes externas de data pueden ser secundarias (provienen de partners con los que has cerrado un acuerdo de cesión / compartición de datos) o bien de terceros (third party data), recogida de un proveedor externo como Amazon o Zeotap.

- Ad verification: Empresas que ofrecen tecnología que permite evitar que la publicidad se difunda en sites poco recomendables. Realizan el seguimiento de las piezas a través de píxeles.

- Brand safe: Proceso que permite garantizar la calidad de la impresión. Herramientas como IAS, MOAT, Double Verify, Fraudlogix o Pixalate nos dan la posibilidad de conocer el "Brand Score" -valoración- de nuestra campaña. Es decir, si nuestra campaña goza o no de "buena salud”. Estas tecnologías nos reportan los sites donde aparezco, visibilidad de las piezas, grado de interacción del público, mapas de calor que indican el lugar exacto en que se produce el clic dentro del banner y una infinidad de métricas.

Tras analizar los agentes que intervienen en la compra programática, conviene analizar los diferentes modelos de compra que existen:

- Subasta abierta / open auction: Subasta donde soporte y anunciante no se conocen. En este caso, el inventario publicitario no está garantizado, siendo la puja a precio variable. En esta modalidad, tienen un papel fundamental las herramientas de brand safe anteriormente reseñadas. 
- Compra programática garantizada (PG): En este caso, el inventario está garantizado y se comercializa a un precio fijo. Esta modalidad es conocida como Deal. Se trata de una compra muy similar a la convencional donde se automatizan ciertos procesos.

- Preferred Deals (PD): Acuerdos privados entre soporte y anunciante a un precio fijo pero con un inventario no garantizado.

- Subasta privada o Mercado Privado (PMP): Punto de intercambio privado donde los soportes venden su inventario, normalmente, en canales verticales (por ejemplo, especializados en viajes o motor) o audiencias. El sistema permite comprar un inventario no garantizado a través de un sistema de pujas a precio variable.

A la hora de activar una campaña en subasta abierta, el primer paso consiste en elegir qué plataforma se va a usar. Una vez decidido este punto se fija la inversión a realizar, el público al que dirigirse y la configuración de black lists y plataformas de brand safety para garantizar la transparencia del proceso. Posteriormente, se determina la categoría de los sites donde se va a insertar la publicidad, así como la audiencia afín. Ya con la campaña en marcha se procede a optimizarla según los datos de respuesta por parte de la audiencia. En el caso de acuerdos privados (deals) el procedimiento varía un poco, pues, es necesario configurar el deal en la plataforma de demanda (DSP).

La implementación de estas tecnologías no está exenta de polémica. Según Papadopoulos et. al. (2017:142) “la recopilación de datos personales de los usuarios se ha vuelto más agresiva y, en algunos casos, incluso intrusiva, generando un enorme debate público en torno a las ventajas de la innovación en publicidad, y los derechos civiles básicos con respecto a la privacidad y la protección personal".

\subsection{La renovación de perfiles profesionales}

La irrupción de las nuevas tecnologías, especialmente internet, ha modificado el sistema mediático. Desde el mundo académico, son muchos los autores que se han interesado por este proceso de cambio y su influencia en el sector publicitario: Castellbanque (2006), Corredor (2011), Del Pino et. al (2014), Garrido, Fernández y Baños (2014), Martín-Guart y Fernández-Cavia (2014).

Para Carcelén, Alameda y Pintado (2017:1664) "la tecnología ha transformado el marketing dentro de las empresas, haciendo que las acciones de marketing digital hayan adquirido un papel predominante en la forma en que tienen las compañías de llegar a los consumidores".

Un fenómeno real y notable, que ha influido notablemente en la publicidad y su estructura, pero que también ha cambiado las expectativas de las marcas. Para Picard (2014:1079) "los mayores anunciantes están hoy constriñendo sus presupuestos publicitarios porque los encuentran menos efectivos que el pasado por temas de retorno e inversión”.

En un entorno cambiante, el Observatorio de la Publicidad de la Asociación Española de Anunciantes (AEA, 2017) señala que el sector de publicidad y estudios de mercado cuenta con 103.600 personas activas en 2016, de los cuales hay 90.000 ocupados y 72.600 asalariados directos. Lo que supone un aumento del $15,6 \%$ respecto al año anterior. 
Si bien los datos de empleo muestran un panorama positivo, la AEA (2017:26) refleja el dinamismo del sector donde "la digitalización, la gestión de contenidos de marca, el big data, la atención de medios propios y medios sociales, están propiciando la aparición de nuevas demandas laborales que se traducen en nuevos ámbitos profesionales".

El Libro Blanco para el diseño de titulaciones universitarias en el marco de la economía digital elaborado por el Ministerio de Industria (2016) sitúa al big data como una de las principales tendencias. La proliferación de datos y su complejidad "demanda soluciones rentables, formas innovadoras de procesamiento de la información, para mejorar su comprensión y la toma de decisiones en las empresas".

El Estudio Top 25 de las profesiones digitales elaborado por INESDI (2018:12-13), señala 9 grandes áreas donde la digitalización tiene especial influencia. Según este informe, desde 2016, en el ámbito del marketing digital se comenzaron a demandar profesionales responsables de conversión y captación programática (RTB Managers). Por otra parte, el segmento del Big Data \& Business Analytics comienza a publicar ofertas desde 2014 buscando perfiles tales como analistas digitales, analistas big data, analistas de inteligencia de cliente o CRM Managers. Según este mismo estudio, desde 2017, comienzan a aparecer ofertas laborales como Responsable de Cuentas Digitales (Digital Account Manager), sin embargo, fuentes del sector afirman que este perfil existe desde, al menos, 10 años antes.

Los resultados del estudio muestran que el $38 \%$ de las ofertas laborales son copadas por el ámbito del marketing digital (INESDI, 2018:15). Sin embargo, se aprecia un descenso motivado por la creciente especialización y el crecimiento de otros segmentos como el contenido digital (+9 puntos porcentuales).

Existe pues la conciencia de que la realidad de la nueva comunicación dibuja un escenario diferente. En el caso de la industria publicitaria, la irrupción de tecnologías como la programática y el big data exigen la convivencia de los clásicos perfiles publicitarios, con otros asociados a competencias digitales que no tienen por qué estar ligados a la comunicación comercial.

Una situación que no debe pasar desapercibida para las universidades que cuentan con estudios de comunicación. Para Wymbs $(2011,2016)$ - citado por Carcelén, Alameda y Pintado (2017:1666) - "la Universidad tiene una necesidad de realizar un profundo cambio en la forma de enseñar disciplinas relacionadas con el marketing y la comunicación, incluyendo asignaturas relacionadas con la era digital y el análisis de datos".

La nueva coyuntura exige, por tanto, el diseño de planes de estudios que guarden cierta coherencia con las demandas del entorno digital, dotando a los estudiantes de herramientas que le permitan trabajar en un mercado laboral cada vez más competitivo.

\section{Métodos}

\subsection{La técnica escogida}

El método Delphi es "una modalidad de investigación evaluativa de enfoque cualitativo, que busca respuestas consensuadas de un grupo de expertos a una serie de cuestiones específicas” (Del Río, 2013: 138). Para Gaitán y Piñuel (1998: 136), 
estos expertos son interrogados sucesivamente, de forma separada, durante una serie de rondas. Esta técnica plantea, según Barranco (1995), una predicción de acontecimientos en el sector en cuestión, la clasificación de estas declaraciones por el investigador y una sucesión de preguntas individuales a cada uno de los expertos, combinadas con información de los otros expertos vía el investigador. Aunque no es propiamente una modalidad conversacional, el método Delphi posee una dinámica dialógica en la que los investigadores tienen una labor mediadora. Para algunos autores, se encuentra a medio camino entre la discusión grupal, la entrevista, e incluso, la encuesta (Ruiz Ispizúa, 1989; Gaitán y Piñuel, 1998: 137). Este método se ha complementado con entrevistas en profundidad a tres expertos de relevancia en el sector. Su participación ha servido para validar los cuestionarios y los resultados obtenidos.

\subsection{Procedimiento}

Tras determinar los objetivos del estudio, la primera tarea consistió en contactar con los expertos. La tarea fue realizada por el grupo coordinador de este estudio, formado por los tres investigadores que lo firman y tres profesionales con amplio bagaje en el ámbito del marketing digital y publicidad programática: Álvaro Cristóbal de Rebold, Gonzalo Galván (BMIND) y Mikel Lekaroz (OZ Digital y Adbibo Tech).

Durante 2 semanas, los investigadores (con ayuda del resto de miembros del grupo coordinador) contactaron vía telefónica e email con diferentes agentes del sector (anunciantes, agencias de medios, soportes y nuevos operadores como DSP's y Trading Desk) y profesores y académicos con investigaciones previas en el ámbito de la publicidad y marketing digital. El criterio de selección de la muestra estaba basado, en primer lugar, en la capacidad y experiencia del entrevistado y, en segundo lugar, se pretendía dar voz a todos los agentes del ecosistema publicitario digital relacionado con la compra programática. Se informó también de la duración aproximada del proceso y el uso de la información. Se motivó a la respuesta explicando los beneficios potenciales del estudio no sólo para el entorno académico, sino también para el ámbito profesional.

Tras la ronda de contactos previos, se procedió al envío de un total de 25 correos electrónicos de invitación al panel de expertos. En esta comunicación se informaba de la actividad a realizar, objetivos de la misma, dinámica de las rondas de preguntas y la confirmación expresa de participación en la investigación. A esta comunicación, respondieron afirmativamente 20 personas, sin embargo, dos de ellas, aunque tenían voluntad de colaborar con el estudio, tuvieron que renunciar debido a las cláusulas de confidencial que tienen firmadas con sus empresas. Conviene aclarar que, la compra programática es un sector emergente, en continuo cambio y donde la movilidad laboral es muy frecuente. Los trabajadores de consultoras y agencias están sometidos a una regulación específica y restrictiva en cuanto a sus declaraciones que ha limitado nuestro estudio. De hecho, algunos de los declarantes que sí aceptaron participar, incidieron en el anonimato de sus respuestas.

Finalmente, la muestra quedó compuesta por 18 profesionales y académicos expertos en publicidad programática. Están representados en este estudio agencias de medios digitales (OMD, Performics - Grupo Publicis - ), agencias especializadas en programática (Rebold, Wise Advertising), trading desk (Havas Programmatic HUB - PMP-, The Trade Desk, Adbibo Tech, BMind), proveedores tecnológicos (DSP-SSP) como Smartclip, AppNexus-Xandr, OZ Digital; anunciantes, soportes. 
consultores independientes y profesores universitarios especializados en marketing digital de la Universidad Francisco de Vitoria y la Universidad Complutense de Madrid.

Tras fijar la muestra se procedió al envío del primer cuestionario, consistente en 5 preguntas abiertas. Conviene señalar que la primera (P.1 - Define la compra programática. Descripción del sistema y diferencias y semejanzas con RTB) no fue utilizada en el Delphi pues perseguía recabar información teórica respecto al objeto de estudio con el propósito de lograr una definición clara y unívoca de "Programmatic Advertising" y sus relaciones con el Real Time Bidding. Por esta razón, siguiendo las recomendaciones de Correyero y Baladrón (2008:66), "se garantizó la confidencialidad de las respuestas de esta parte del cuestionario, comprometiéndose los investigadores a no utilizarlas en fases posteriores del proceso iterativo".

Cuadro 2: Listado de preguntas del primer cuestionario

\begin{tabular}{|c|c|c|}
\hline No & Pregunta & Objetivo \\
\hline 1 & $\begin{array}{l}\text { Define la compra programática. Descripción del sistema y diferen- } \\
\text { cias y semejanzas con RTB }\end{array}$ & $\begin{array}{l}\text { Pregunta exploratoria. Se busca uniformizar la defini- } \\
\text { ción de compra programática y su diferenciación con el } \\
\text { RTB }\end{array}$ \\
\hline 2 & $\begin{array}{l}\text { ¿Cuál es la evolución de la compra programática en España con } \\
\text { respecto al resto de mercados? }\end{array}$ & $\begin{array}{l}\text { Conocer evolución del mercado español vs resto del } \\
\text { mundo }\end{array}$ \\
\hline 3 & $\begin{array}{l}\text { En el campo de la compra programática ¿Qué perfiles profesionales } \\
\text { se están demandando? }\end{array}$ & Conocer demanda de profesionales en el sector \\
\hline 4 & $\begin{array}{l}\text { La formación que se imparte en Grados Univesitarios en Comuni- } \\
\text { cación (Publicidad, Comunicación Audiovisual, etc.) ¿Es adecuada } \\
\text { para el desempeño de las tareas requeridas en la creación y gestión } \\
\text { de campañas de Publicidad Programática? Además de egresados } \\
\text { en Comunicación, ¿Qué otro tipo de estudiantes tienen cabida en } \\
\text { la Publicidad Programática? }\end{array}$ & $\begin{array}{l}\text { Conocer el grado de adaptación de los grados universita- } \\
\text { rios a las demandas de la programática. }\end{array}$ \\
\hline 5 & $\begin{array}{l}\text { ¿Cuál es el futuro del sector en los próximos años? ¿De qué manera } \\
\text { se redefine la función del planificador de medios tradicional? }\end{array}$ & $\begin{array}{l}\text { Hacer prospectiva sobre el futuro del sector y el rol del } \\
\text { planificador de medios tradicional }\end{array}$ \\
\hline
\end{tabular}

Fuente: Elaboración propia

El primer cuestionario fue analizado pormenorizadamente por el grupo coordinador, encontrando el consenso en una de las 4 preguntas que se aplicaron en el Delphi. Como ya hemos señalado, la pregunta 1 (P.1) tenía un carácter exploratorio, donde el objetivo era tratar de obtener una definición clara de algunos conceptos clave relacionados con el fenómeno a analizar. Las respuestas a la pregunta 3 (P.3) alcanzaron un grado de consenso muy alto tal y como señalaremos en la exposición de resultados.

En la segunda ronda, se seleccionó a la muestra de expertos completa. En esta fase se formulaban de nuevo las tres preguntas que habían suscitado mayor nivel de disenso entre los participantes (P.2, P.4 y P.5). Ahora bien, en esta ocasión, se optó por respuestas cerradas a preguntas dicotómicas (Sí/No) como el caso de la P.2.1, la posibilidad de escoger entre varias opciones de respuesta mediante casillas de verificación (P.2.2) y la valoración de escalas (de 1 -muy en desacuerdo-a 5 -muy de acuerdo-) como el caso de las preguntas P.2.2, P. 4.1, P. 4.2, P.4.3 y de la P.5.1 a la P.5.6. 
Un total de 14 expertos devolvió sus respuestas a la segunda ronda.

Cuadro 3: Listado de preguntas del segundo cuestionario

\begin{tabular}{|c|c|c|}
\hline No & Pregunta & Cuestiones específicas \\
\hline 2 & $\begin{array}{l}\text { A la pregunta, ¿Cuál es la evolución de } \\
\text { la compra programática en España con } \\
\text { respecto al resto de mercados? El grupo } \\
\text { ha reflejado diferentes aportaciones. Para } \\
\text { tratar de ahondar en esta cuestión y obtener } \\
\text { resultados que ayuden a fijar una visión } \\
\text { futura y consensuada del sector, planteamos } \\
\text { dos cuestiones adicionales. }\end{array}$ & $\begin{array}{l}2.1 \text { ¿Consideras que la evolución del negocio de la compra programática } \\
\text { está siendo muy rápido en España? Escala lineal de } 1 \text { (Muy en desacuerdo) } \\
\text { a } 5 \text { (Muy de acuerdo) } \\
2.2 \text { Señale las posibles causas del lento crecimiento de la compra } \\
\text { programática en España frente a otros países. Entre las posibles causas } \\
\text { se podía escoger entre: no transparencia en la negociación, desconfianza } \\
\text { en el inventario, falta de formación en programática, falta de innovación } \\
\text { y estrategia en anunciantes y medios, costes tecnológicos, factores } \\
\text { económicos, calidad de la data y acuerdos directos entre agencias y } \\
\text { anunciantes que dificultan el desarrollo de la programática. }\end{array}$ \\
\hline 4 & $\begin{array}{l}\text { A la pregunta, "La formación que se } \\
\text { imparte en Grados en Comunicación, ¿Es } \\
\text { adecuada para el desempeño de las tareas } \\
\text { requeridas en la creación y gestión de } \\
\text { campañas de Publicidad Programática?". } \\
\text { El panel responde de manera unánime que } \\
\text { la formación es nula o prácticamente nula. } \\
\text { Sí existe formación específica en programas } \\
\text { y cursos especializados donde profundizar. } \\
\text { Ante esta situación y si fueses un gestor } \\
\text { educativo del área de comunicación de un } \\
\text { grado universitario, ¿Cómo incorporarías la } \\
\text { formación en programática? }\end{array}$ & $\begin{array}{l}\text { 4.1 Como materia obligatoria en el plan de estudios. Escala lineal de } 1 \text { (Muy } \\
\text { en desacuerdo) a } 5 \text { (Muy de acuerdo). } \\
4.2 \text { Como materia optativa en el plan de estudios. Escala lineal de } 1 \text { (Muy en } \\
\text { desacuerdo) a } 5 \text { (Muy de acuerdo). } \\
4.3 \text { No es formación propia de un grado en comunicación, debería } \\
\text { impartirse en grados como informática, ingeniería o business analytics. } \\
\text { Escala lineal de } 1 \text { (Muy en desacuerdo) a } 5 \text { (Muy de acuerdo). }\end{array}$ \\
\hline 5 & $\begin{array}{l}\text { A la pregunta, “¿Cuál es el futuro del sector } \\
\text { en los próximos años?" Existe consenso en } \\
\text { cuanto a que se producirá una expansión a } \\
\text { otros medios y dispositivos. En la segunda } \\
\text { parte de la pregunta, ¿De qué manera se } \\
\text { redefine la función del planificador de medios } \\
\text { tradicional? Estimamos oportuno concretar } \\
\text { qué aspectos se deben fortalecer para una } \\
\text { efectiva reconversión. }\end{array}$ & $\begin{array}{l}\text { 5.1. Formación específica centrada en analítica y gestión de la data. } \\
\text { 5.2. Tareas comerciales con amplio conocimiento del ecosistema } \\
\text { programático. } \\
\text { 5.3. Tareas de optimización de campañas. } \\
\text { 5.4. Especialización en gestión del Ad Server y traficado de creatividades } \\
\text { 5.5. Integración en equipos mixtos formados por publicitarios o expertos en } \\
\text { marketing y tecnólogos. } \\
\text { 5.6. No hay posibilidad de reciclaje, deben reorientar su carrera profesional } \\
\text { En todas las opciones la respuesta es mediante escala lineal de } 1 \text { (Muy en } \\
\text { desacuerdo) a } 5 \text { (Muy de acuerdo). }\end{array}$ \\
\hline
\end{tabular}

Fuente: Elaboración propia

Tras la aplicación de la ronda 2, se analizaron los resultados hallando consenso en la mayoría de cuestiones planteadas. De ahí, que se procediera a finalizar el cuestionario. 


\section{Resultados}

Tras haber expuesto el estado de la cuestión, objetivos e hipótesis y justificar el objeto de estudio. Y tras haber detallado la metodología utilizada incluyendo la técnica, tamaño muestral, trabajo de campo y el procesamiento de los datos obtenidos, vamos a presentar los resultados más destacables.

\section{1. (P.1) Define la compra programática, Descripción del sistema y diferencias y semejanzas con RTB}

Como mencionamos en el apartado métodos, la P.1 no está enmarcada dentro de la metodología escogida pero, las respuestas de los miembros del panel de expertos, nos permiten abordar una necesaria definición del fenómeno y su papel en el ecosistema publicitario.

La mayoría de miembros del panel coinciden er que la programática es un sistema automatizado de compra de espacios publicitarios. Originariamente, el proceso estaba concebido para medios y soportes online, pero actualmente, está desembarcando en medios considerados como tradicionales como la radio o la televisión.

La emergencia del modelo surge del paso de un enfoque de compra basado en la cantidad de audiencia afín, alcanzada e impactada (site centric), a un enfoque donde el usuario es el centro del proceso (user centric). En este segundo enfoque, la data juega un papel fundamental, pues no sólo identifica un perfil de usuario y su comportamiento, sino que ayuda a personalizar el mensaje del anuncio y el contenido que se muestra.

Los expertos coinciden en que existe cierta confusión terminológica en cuando a los tipos de enfoque de la compra programática. El Real Time Bidding o Subasta en Tiempo Real (RTB) es uno de los modos de compra programática, pero no el único.

Esta confusión surge porque el RTB es la modalidad más frecuente y antigua. Sin embargo, sólo hace referencia a un inventario subastado. Si el inventario publicitario no es subastado, se llama programática directa y existen dos modalidades: garantizado (programmatic guaranteed o PG) y, si no es garantizado, se denomina Preferred Deal (PD).

\section{2. (P.2) ¿Cuál es la evolución de la compra programática en España con respecto al resto de mercados?}

No existe consenso en la primera ronda de preguntas, de manera que, en la ronda 2, se amplía con dos cuestiones. 
3.2.1. ¿Consideras que la evolución del negocio de la compra programática está siendo muy rápido en España?

Cuadro 4: Estadísticos descriptivos de la pregunta (P. 2.1)

\begin{tabular}{|c|c|c|c|}
\hline Pregunta & Mediana & Media & Desviación Típica \\
\hline $\begin{array}{l}\text { ¿Consideras que la evolución del negocio de la compra } \\
\text { programática está siendo muy rápido en España? }\end{array}$ & 2 & 2,21 & 0,93 \\
\hline
\end{tabular}

Fuente: Elaboración propia

Teniendo en cuenta que el valor 1 equivale a muy en desacuerdo, y el 5 a muy de acuerdo. El resultado obtenido nos permite concluir que el panel de expertos considera que el desarrollo de la publicidad programática en España no ha alcanzado aún su madurez.

\subsubsection{Señale las posibles causas del lento crecimiento de la compra programática en España frente a otros países.}

Existían 8 opciones de respuesta. Escogidas tras el análisis de las respuestas a la P.2 en la primera ronda de no consenso. Tras el análisis a las respuestas de la segunda ronda, más específica, estos son los resultados:

- No transparencia en la negociación $(63,7 \%)$

- Desconfianza en el inventario $(45,5 \%)$

- Falta de formación en programática (100\%)

- Falta de innovación y estrategia de las agencias de medios tradicionales y los grandes grupos mediáticos (45,5\%)

- Costes tecnológicos $(18,2 \%)$

- Factores económicos $(18,2 \%)$

- Calidad de la data $(27,3 \%)$

- Acuerdos directos entre agencias y publishers (27,3\%)

Para los panelistas, la principal razón del lento crecimiento de la compra programática en España es la falta de formación específica en la disciplina. La segunda es la ausencia de transparencia en la negociación. Por otra parte, los expertos comentan que existe desconfianza en el inventario y falta de innovación y estrategia de las agencias de medios tradicionales y los grandes grupos mediáticos, dueños de los soportes.

\section{3. (P.3) En el campo de la compra programática ¿Qué perfiles profesionales se están demandando?}

El panel de expertos coincide en señalar que el en campo de la publicidad programática son necesarios perfiles analíticos que no sólo entiendan cómo manejar una plataforma (traders) sino también analistas de datos, cuyas responsabilidades estén enfocadas en la recogida, uso y explotación de la data y optimización de herramientas como DMP’s.

Además, se precisa de informáticos y programadores que ayuden a las integraciones entre diferentes plataformas. 
3.4. La formación que se imparte en Grados Universitarios en Comunicación (Publicidad, Comunicación Audiovisual, etc.) ¿Es adecuada para el desempeño de las tareas requeridas en la creación y gestión de campañas de Publicidad Programática? Además de egresados en Comunicación, ¿Qué otro tipo de estudiantes tienen cabida en la Publicidad Programática?

El panel responde de manera unánime que la formación actual en grados universitarios es nula o prácticamente nula en publicidad programática. Declarando que sí existe formación específica en programas y cursos especializados puestos en marcha por escuelas de negocios y centros de posgrado. Por esta razón, se formuló una nueva cuestión en la segunda ronda que permitiera profundizar en la posible integración / o no de la formación en programática en los grados universitarios de comunicación y qué tipo de formación debería ser.

De esta manera, se planteó la posibilidad de convertirla en materia obligatoria, optativa o eliminarla de la formación propia de un grado en comunicación.

Antes estas cuestiones, los panelistas recalcaron que la formación debería incluirse en los planes de estudios, al menos, como materia optativa que permita al alumno una formación básica en esta disciplina.

\section{5. ¿Cuál es el futuro del sector en los próximos años? ¿De qué manera se redefine la función del planificador de medios tradicional?}

En la primera ronda, se logró el consenso en cuanto a que se producirá una expansión notable de la publicidad programática, especialmente, que vendrá de la exportación del modelo a otros medios y dispositivos. En la segunda parte de la pregunta, estimamos oportuno concretar qué aspectos se deben fortalecer en la formación de los planificadores de medios para lograr una efectiva formación / reconversión. Para ello, se decidió plantear al panel una serie de afirmaciones a las que debían asignar una puntuación en una escala lineal.

\subsubsection{Formación específica centrada en analítica y gestión de la data}

El 54\% de los panelistas afirmaron estar muy de acuerdo con la necesidad de formación centrada en analítica y gestión de grandes volúmenes de datos.

\subsubsection{Tareas comerciales con amplio conocimiento del ecosistema programático}

El 72,8\% de miembros del panel afirman estar de acuerdo o muy de acuerdo con esta afirmación.

\subsubsection{Tareas de optimización de campañas}

La amplia mayoría de expertos respondieron que era absolutamente necesaria formación específica en optimización de campañas en programática.

\subsubsection{Especialización en gestión del Ad Server y traficado de creatividades}

Existe cierta dispersión en las respuestas a esta cuestión. Prevaleciendo el grado de acuerdo (54,5\%) en la necesidad de conocer los procesos de trabajo en el adserver y traficado de creatividades. 


\subsubsection{Integración de equipos mixtos formados por publicitarios y tecnólogos}

La amplia mayoría de expertos abogaron por la integración de perfiles tecnológicos (informáticos, programadores, analistas) con publicitarios.

\subsubsection{No hay posibilidad de reciclaje, deben reorientar su carrera profesional}

Existe una amplia mayoría de panelistas (72,7\%) que afirman estar muy en desacuerdo con la anterior afirmación.

\subsection{Conclusiones finales del estudio}

La programática más que la automatización de una tarea manual, representa una nueva forma de planificar medios en un entorno online. Su avance es producto de la confluencia de varios factores, entre ellos, el aumento de la velocidad de conexión y la agilidad de las computadoras en gestionar grandes volúmenes de datos.

Se trata de un ecosistema muy complejo, en ocasiones difícil de entender, por el enorme número de players y tecnologías que intervienen: Trading Desks, plataformas del lado de la demanda (DSP’s), Ad Exchanges, proveedores de data, plataformas ligadas a la parte de soporte (SSP's), redes, mercados privados, publishers, etc.

Este entramado de actores garantiza la eficiencia de un sistema que, en el pasado, funcionaba con otras dinámicas. En la planificación digital tradicional, el enfoque se centraba en la cantidad de audiencia afín alcanzada en un determinado soporte (ej: medio de comunicación). La audiencia estaba asociada a un canal determinado lo que implicaba la pérdida de impactos fuera de nuestro target. El método de búsqueda de usuarios era poco preciso, escogiendo sites con mucha cobertura (ej: portales de internet), o bien, sites específicos (nicho).

Es importante diferenciar compra programática de Real Time Bidding. El RTB es un modelo de compra en el que, a través de una serie de variables establecidas en la plataforma del lado de la demanda (DSP), se accede a pujar por determinadas impresiones en tiempo real. Ahora, esta modalidad, puede convivir con otras ya mencionadas en el estudio, como la compra garantizada $(\mathrm{PG})$.

El sector de la publicidad programática crece año a año en nuestro país. Sin embargo, las cifras de inversión no superan el $20 \%$ frente a otros mercados como Reino Unido, Alemania o Francia.

Tras consultar a los expertos del panel, la mayoría coincide en la falta de formación específica como una de las principales causas del escaso desarrollo de esta modalidad publicitaria. Se trata de un sector en ebullición, pero donde falta mano de obra. De acuerdo con Carcelén, Alameda y Pintado (2017: 1650), en determinadas profesiones relacionadas con lo digital, existe un gap entre lo que las empresas demandan y la preparación y formación de los profesionales que acceden al mundo laboral.

La Universidad, poco a poco, va adaptando sus planes de estudios a las nuevas necesidades. Aunque, en este sentido, las escuelas de negocio y centros de posgrado cuentan con mayor flexibilidad en la presentación de oferta, debido a que, en muchos casos, no se trata de estudios oficiales cuyo itinerario formativo ha de validarse en el ministerio correspondiente, requerimiento por el que pasan todas las Universidades que pretenden lanzar títulos oficiales de grado. 
Otra causa del escaso desarrollo de la programática es la falta de transparencia en la negociación, probablemente derivada de la gran cantidad de agentes que intervienen en el proceso, y que, de una manera u otra, aumentan los costes. También existe cierta desconfianza en el inventario, ya que, en un primer momento, los espacios publicitarios orientados a la venta automatizada eran aquellos que eran invendibles a través de otros canales. Actualmente los procesos han cambiado, sin embargo, existe cierta reticencia de los grandes grupos de comunicación a ofertar todo su inventario en programática debido a que obtienen más ingresos y de manera más rápida a través de otras vías. La progresiva implementación de herramientas que garantizan la visibilidad, aporta seguridad a las marcas contra el fraude y miden la rentabilidad de las inversiones ayudarán al desarrollo del sector.

De hecho, se prevé una expansión notable de la publicidad programática en los próximos años. Un crecimiento que vendrá de la mano de la exportación del modelo aplicado en Internet a otros medios (televisión, radio, exterior, etc.) y dispositivos.

Teniendo en cuenta que hemos pasado de hablar de impresiones a hablar de audiencias, el planificador del futuro debe saber generar un plan. Ha de detectar la confluencia de la programática con otras acciones como el marketing de buscadores (SEM) o acciones convencionales. De ahí que tenga que recibir formación específica centrada en analítica y gestión de la data, optimización de campañas, gestión del adserver y traficado de creatividades. Ahora bien, en estas primeras fases de implantación, el planificador debe realizar una tarea de evangelización, ampliando sus dotes comerciales para vender tecnología sin caer en un vocabulario vacío y lleno de tecnicismos que, en muchas ocasiones, el cliente no entiende.

El sector es consciente del proceso de cambio, especialmente el ámbito de los planificadores de medios digitales, que van a requerir de nuevas habilidades y conocimientos para realizar su actividad. A día de hoy los perfiles que mejor ejecutan y plasman estrategias de programática son aquellos que pueden interpretar las planificaciones en un adserver, ejecutarlas en un DSP, integrar audiencias en un DMP y optimizarlas. Si bien, a medio y largo plazo, se aboga por la integración de perfiles tecnológicos con publicitarios creando duplas donde se una, el pensamiento analítico de unos, con el know how de las herramientas y procesos de la publicidad online de otros.

\section{Referencias bibliográficas}

Alhabash, S., Mundel, J., \& Hussain, S. A. (2017). "Social media advertising. Unraveling the mistery box", En Rodgers, S. y Thorson, E. (eds) Digital Advertising: Theory and Research, New York: Taylor \& Francis, pp. 285-298.

Álvarez-Flores, Erika P.; Núñez-Gómez, Patricia; Olivares-Santamarina, José P. (2018). "Perfiles profesionales y salidas laborales para graduados en Publicidad y Relaciones públicas: de la especialización a la hibridación". El profesional de la información, v. 27, n. 1, pp. 136-147.

Boone, G., Secci, J., \& Gallant, L. (2010). Emerging trends in online advertising. Doxa communicacion, n. 5, pp. 241-253.

Carcelén, S. Alameda, D. y Pintado, T. (2017). Prácticas, competencias y tendencias de la comunicación publicitaria digital: una visión desde la perspectiva de los anunciantes españoles. Revista Latina de Comunicación Social, (72), pp. 1648-1669.

Carlson, M. (2015). When news sites go native: Redefining the advertising-editorial divide in response to native advertising. Journalism, 16(7), 849-865. 
Carnés, I. (2018, 23 de septiembre). Entrevista con Nacho Carnés [Correo electrónico].

Carrillo-Durán, M. V., \& Rodríguez-Silgado, A. (2018). El ecosistema programático. La nueva publicidad digital que conecta datos con personas. El profesional de la información, 27(1), 195-201.

Castellblanque, M. (2006). Perfiles profesionales de publicidad y ámbitos afines: ¿qué quiero ser?¿ qué quiero hacer?:̇ para qué tengo talento? (Vol. 64). Editorial UOC.

Caumont, S., Kandjian, F., \& Talazac, F. (2013). Google AdWords: la guía completa: optimice sus campañas para obtener más ingresos. Ediciones ENI.

Chatterjee, P. (2008). Are unclicked ads wasted? Enduring effects of banner and pop-up ad exposures on brand memory and attitudes. Journal of electronic commerce Research, 9 (1).

Cho, C. H., Lee, J. G., \& Tharp, M. (2001). Different forced-exposure levels to banner advertisements. Journal of Advertising Research, 41(4), pp. 45-56.

Cook, K. (2016). "A brief history of online advertising". Hubspot.com. En: https://blog.hubspot.com/marketing/history-of-online-advertising [Consultado el 05/07/2018].

Corredor, P. (2011). “En clave digital: nuevos profesionales en publicidad”. Telos: Cuadernos de comunicación e innovación, n. 87 , pp. $97-100$.

Correyero Ruiz, B., \& Baladrón Pazos, A. (2008). Las revistas profesionales especializadas en publicidad en España: resultados de un estudio Delphi. Doxa comunicación, 7.

Cristóbal, A. (2018, 4 de Septiembre). Entrevista con Álvaro Cristóbal [Correo electrónico].

Del Pino, A. , Castelló, A. , Ramos, I. (2013): La comunicación en cambio constante. Madrid: Fragua.

Del Río, D. (2013). Diccionario-glosario de metodología de la investigación social. Madrid: Editorial UNED.

Diao, F., \& Sundar, S. S. (2004). Orienting response and memory for web advertisements: Exploring effects of pop-up window and animation. Communication research, 31(5), pp. 537-567.

Edwards, S. M., Li, H., \& Lee, J. H. (2002). Forced exposure and psychological reactance: Antecedents and consequences of the perceived intrusiveness of pop-up ads. Journal of Advertising, 31(3), pp. 83-95.

Garrido, P., Fernández-Fernández, P., \& Baños-González, M. (2014). Estrategias ante la nueva situación tecnológica y económica del sector publicitario español. Revista Mediterránea de Comunicación, v. 5, n. 2.

IAB (2004). Glosario de Términos IAB Spain (Primera Parte). Recuperado de https://goo.gl/Q5ciWA

IAB (2004). Glosario de Términos IAB Spain (Segunda Parte). Recuperado de https://goo.gl/tk4uZC

IAB (2014). Libro Blanco de Compra Programática. Recuperado de https://goo.gl/j9fd67

IAB (2015). Guía Legal sobre Publicidad Nativa. Recuperado de https://goo.gl/1wVzrp

Jansen, B. J., \& Mullen, T. (2008). Sponsored search: an overview of the concept, history, and technology. International Journal of Electronic Business, 6(2), pp. 114-131. 
Maceiras, A. (2018) “No sin brand safe”. Bmind Website. En: https://www.bmind.es/es/no-sin-brand-safe/ [Consultado el $27 / 10 / 2018]$.

Madinaveitia, E. (2018) [Bit Audiovisual]. (2018, junio, 12). Jornadas Bit Audiovisual 2018. Publicidad programática. [Archivo de vídeo] Recuperado de https://youtu.be/acfQYAfuBVc

Martín-Guart, R. F., \& Fernández Cavia, J. (2014). La publicidad y la agencia de medios frente al cambio en el ecosistema mediático. Cuadernos. info, (34), 13-25.

Medoff, N. J., \& Kaye, B. (2016). Electronic media: then, now, and later. New dYork: Taylor \& Francis.

King, B. (2012) Bank 3.0: Why banking is no longer somewhere you go but something you do. Singapore: John Wiley \& Sons.

Malthouse, E. C., Maslowska, E., \& Franks, J. U. (2018). Understanding programmatic TV advertising. International Journal of Advertising, 1-16.

O’Dell, A. To Do Programmatic Advertising Yourself, Start by Picking a DSP. E-content Mag. En: http://www.econtentmag. com/Articles/Editorial/Industry-Insights/To-Do-Programmatic-Advertising-Yourself-Start-by-Picking-a-DSP-117193. htm [Consultado el 28/10/2018].

Orense, M., \& Rojas, O. (2010). SEO. Cómo triunfar en buscadores. Madrid: ESIC.

Papadopoulos, P., Rodriguez, P. R., Kourtellis, N., \& Laoutaris, N. (2017, November). If you are not paying for it, you are the product: How much do advertisers pay to reach you?. In Proceedings of the 2017 Internet Measurement Conference, pp. 142156. ACM.

Fajula, A. \& Roca, D. (2000). "El papel del publicitario ante un nuevo contexto tecnológico". Revista Latina de Comunicación Social, vol. 3 (25). Recuperado el 12 de septiembre de 2018 de http://www.ull.es/publicaciones/latina/aa2000yen/141vadavid.html

Picard, R. (2014). Las industrias informativas: ¿tienen futuro?. Palabra Clave, 17(4), pp. 1069-1096.

Rodríguez, M. (2017) [CreaTECH540]. (2017, junio, 10). Publicidad programática, una visión holística. [Archivo de vídeo] Recuperado de https://youtu.be/96dBamz4hpE

Sánchez, M. y De Frutos, B. (2007) “Recursos creativos de la publicidad en internet”. Doxa Comunicación. Revista interdisciplinar de estudios de Comunicación y Ciencias Sociales, 2007, n. 5, pp. 159-188.

Said, M. (2014). “The evolution of online advertising”. Mediamath.com. En: http://www.mediamath.com/blog/the-evolution-online-advertising/ [Consultado el 20/08/2018].

Schmid, D. (2017). “The History of Programmatic Advertising: Everything You Need to Know”. DisruptorDaily.com, En: https://www.disruptordaily.com/the-history-of-programmatic-advertising-everything-you-need-to-know/ [Consultado el $22 / 09 / 2018]$. 
